\section{Polyalanine expansion and frameshift mutations of the paired-like homeobox gene PHOX2B in congenital central hypoventilation syndrome}

Published online 17 March 2003, doi:10.1038/ng1130

Congenital central hypoventilation syndrome (CCHS or Ondine's curse; OMIM 209880) is a life-threatening disorder involving an impaired ventilatory response to hypercarbia and hypoxemia. This core phenotype is associated with lower-penetrance anomalies of the autonomic nervous system (ANS) including Hirschsprung disease and tumors of neural-crest derivatives such as ganglioneuromas and neuroblastomas. In mice, the development of ANS reflex circuits is dependent on the paired-like homeobox gene Phox $2 b$. Thus, we regarded its human ortholog, $P H O X 2 B$, as a candidate gene in CCHS. We found heterozygous de novo mutations in PHOX2B in 18 of 29 individuals with CCHS. Most mutations consisted of 5-9 alanine expansions within a 20-residue polyalanine tract probably resulting from non-homologous recombination. We show that $P H O X 2 B$ is expressed in both the central and the peripheral ANS during human embryonic development. Our data support an essential role of PHOX2B in the normal patterning of the autonomous ventilation system and, more generally, of the ANS in humans.

$a$

$b$

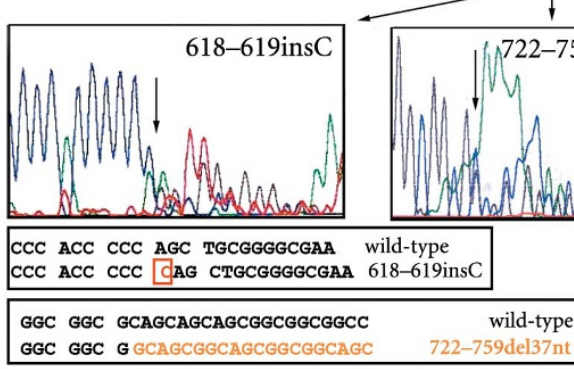

A genetic origin of $\mathrm{CCHS}^{1}$ has long been suspected based on concordance in monozygotic twins ${ }^{2}$, rare familial cases (siblings, half-siblings, and mother-tochild transmission $)^{3}$ and segregation analysis suggesting an autosmal dominant locus with low penetrance or a multigenic model ${ }^{4}$. So far, only lowpenetrant predisposing mutations of the RET-Glial cell line-derived neurotrophic factor (GDNF), endothelin 3 (EDN3) and brain-derived neurotrophic factor (BDNF) pathways have been reported in a few individuals with $\mathrm{CCHS}^{5-7}$. Considering the broad range of defects in the ANS in CCHS on the one hand and the key role of Phox $2 b$ in the ontogeny of the ANS reflex circuits in mice ${ }^{8,9}$ on the other hand, we regarded $P H O X 2 B$ (also called $P M X 2 B$ and $N B P H O X$ ) as a candidate gene in the disease.

PHOX2B maps to chromosome $4 \mathrm{p} 12$ and encodes a highly conserved homeobox transcription factor of 314 amino acids with two short and stable polyalanine
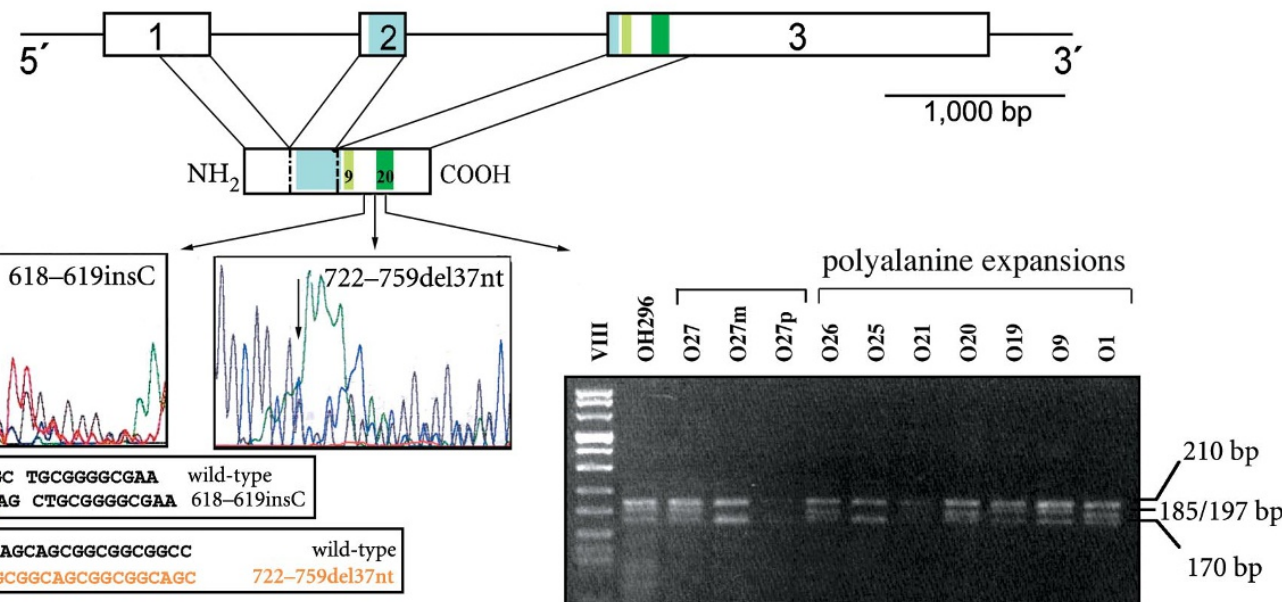

c

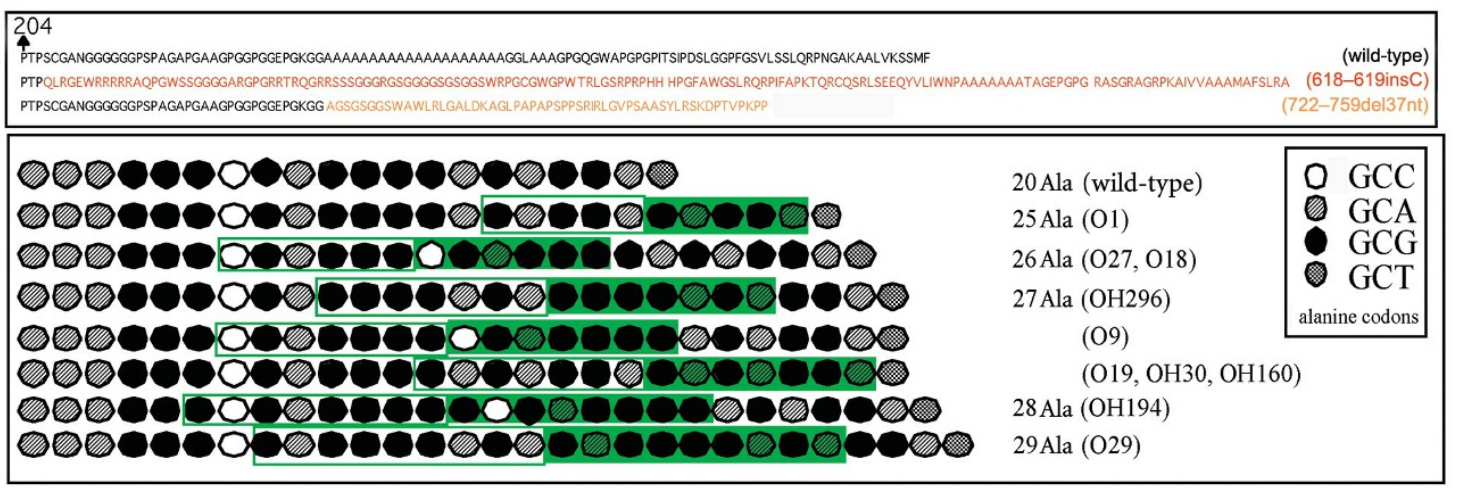

Fig. 1 Mutations of $P H O X 2 B$ in CCHS. a, Genomic organization of $P H O X 2 B$ and schematic representation of the PHOX2B protein. The homeobox domain and the 9- and 20-residue polyalanine tracts are indicated (blue and green boxes, respectively). $\boldsymbol{b}$, DNA sequence electropherogram and PCR digestion showing the identified mutations in PHOX2B. Left, Heterozygous frameshift mutations. Right, Heterozygous alanine triplet expansions. Mutated alleles are seen as extra bands of variable size between the 170- and 210-bp bands after enzymatic digestion with Stul of the 380-bp PCR product of PHOX2B exon 3 and migration on a $3 \%$ agarose gel (see Supplementary Note 1 online). c, Mutant PHOX2B proteins. Top, 618-619insC and 722-759del37nt frameshift mutations. Bottom, various polyalanine expansions observed. The duplicated codons are shown in green boxes. 
repeats of 9 and 20 residues, respectively (Fig. 1a). The length of the polyalanine tracts is conserved in mice and humans. We screened the coding sequence of $P H O X 2 B$ in a series of 29 unrelated individuals with CCHS (see Supplementary Note 1 online). Direct DNA sequencing showed heterozygosity with respect to PHOX2B variations in 18 of 29 cases. In 16 of 18 cases, the nucleotide variation was a triplet expansion of 15-27 nucleotides (nt 721-780; Fig. $1 b$ and Supplementary Table 1 online) adding 5-9 alanines to the 20residue polyalanine tract (Fig. 1c). Most mutant genotypes were different, suggesting that they derived from independent mutational events (Fig. 1c). In addition, whenever parents were available, we found that the polyalanine triplet expansion occurred de novo, supporting its role in the disease phenotype (see Supplementary Table 1 online). In two other CCHS cases, a de novo cytosine insertion in a stretch of four cytosines (618-619insC) and a deletion of 37 nucleotides (722-759del37nt)
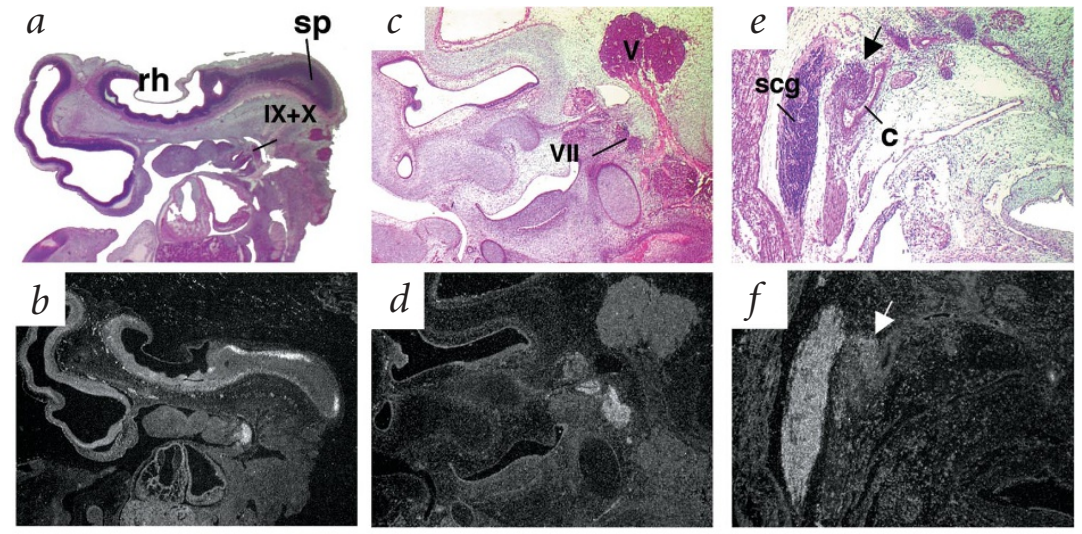

homeobox, predicting a mutant protein with no known function or homology (Fig. 1b,c). The 722-759del37nt mutation could be regarded as an out-of-frame contraction of the polyalanine tract. We found neither alanine triplet expansions nor frameshift mutations in 250 control chromosomes from various ethnic backgrounds. Notably, 2 of 250 control alleles had a small polyalanine contraction ( 5 and 6 triplets, data not shown), suggesting an unequal crossing-over during meiosis rather than a polymerase slippage during replication. In the former case, polyalanine expansions and contractions are equally likely to occur; $P H O X 2 B$ contractions were found as a rare variant in controls.

We found that 2 of 29 individuals with CCHS carried heterozygous variants in genes involved in the same developmenta pathway, namely RET and GDNF (aminoacid substitutions P1039L and R93W, respectively; see Supplementary Table 1 online; ref. 5), in addition to polyalanine
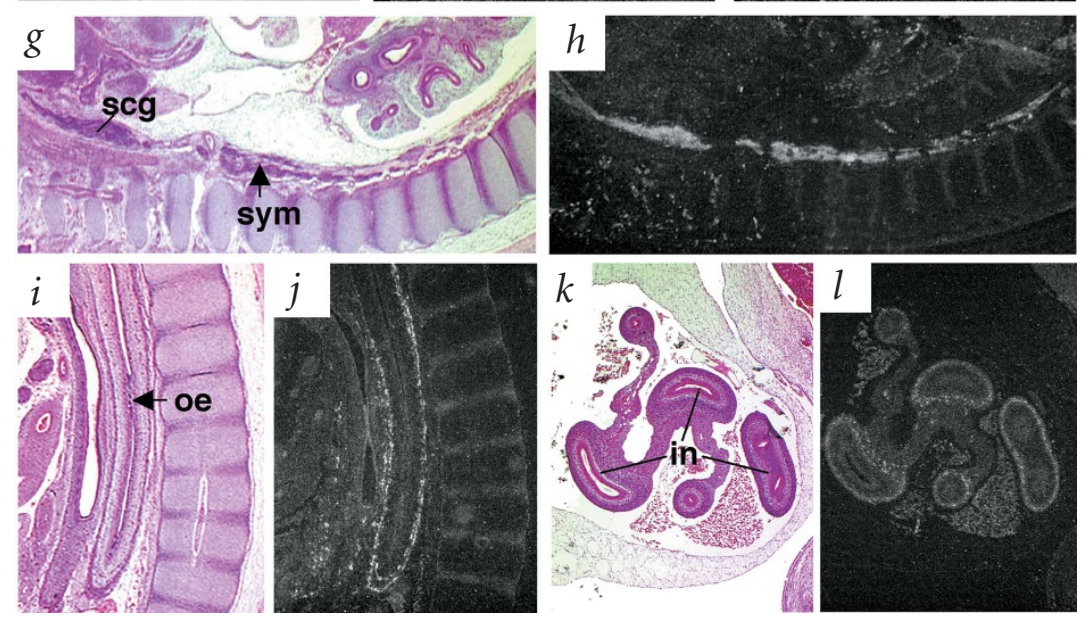

Fig. 2 PHOX2B gene expression in developing human brainstem and enteric nervous system. Slides stained with hematoxylin and eosin $(a, c, e, g, i, k)$ and dark-field illumination of the hybridized adjacent sections $(b, \boldsymbol{d}, \boldsymbol{f}, \boldsymbol{h}, \boldsymbol{j}, \boldsymbol{l})$ at day $33(a, b)$, day $54(c-f)$ and day 47 of development $(g-l)$. Parasagittal sections showing expression of PHOX2B in rhombencephalon (rh; $a, b)$, cervical spinal cord (sp; $a, b)$, seventh (VII) ganglia and ninth/tenth (IX+X) ganglionic complex $(a-d)$, presumptive enteric ganglia of oesophagus (oe) and intestine (in; $i-l)$ and paravertebral sympathetic ganglia $(s y m)$, including the superior cervical ganglion $(s c g ; g, h)$ PHOX2B expression was also detected in the presumptive carotid body (arrow) at the carotid (c) bifurcation, ventral to the superior cervical ganglion $(e, f)$. Magnifications: $a, b, g, h, \times 15 ; c, d, \times 25 ; i-l, \times 30 ; e, f, \times 40$. expansions in $P H O X 2 B$. Notably, Phox2 genes control Ret expression in both sympathetic and enteric neurons in mice ${ }^{8}$. Unlike the polyalanine expansions, however, these variants are neither necessary (most individuals with CCHS do not have any RET or GDNF gene variant) nor sufficient for the disease to occur (carrier parents have no phenotypic expression). These data suggest that $P H O X 2 B$ is the primary disease locus in CCHS. Moreover, we found mutations in $\mathrm{PHOX} 2 \mathrm{~B}$ not only in isolated cases of CCHS but also in individuals with a more complex neural-crest involvement including CCHS and Hirschsprung disease (Haddad syndrome) as well as early-onset neuroblastoma. We did not find any correlation between the size of the polyalanine tract and the complexity of the disease in our analyses.

To confirm the involvement of mutations in $P H O X 2 B$ in the disease phenotype, we studied the expression pattern of $P H O X 2 B$ in early human development (see Supplementary Note 1 online). In accordance with the wide spectrum of ANS dysfunction observed in individuals with CCHS, the expression pattern of $P H O X 2 B$ involved both central autonomic circuits and peripheral neuralcrest derivatives. From day 32 of development, $P H O X 2 B$ was expressed in the seventh ganglion and the ninth/tenth ganglionic complex (Fig. $2 a-d$ ). From day 33 of development, we observed strong expression of $P H O X 2 B$ in terminal rhombomeres $4-8$, in the presumptive enteric ganglia and in the sympathetic chain ganglia (Fig. 2g-l). Finally, we detected expression of $P H O X 2 B$ in the presumptive carotid body at the carotid bifurcation, ventral to the superior cervical ganglion, which also expressed $\mathrm{PHOX} 2 \mathrm{~B}$ (Fig. 2e,f). We did not detect any $P H O X 2 B$ expression in adrenal medulla at the stages investigated.

Polyalanine expansion mutations involving homeodomain or non-homeodomain transcription factors (HOXA13, HOXD13, ARX, RUNX2, ZIC2, FOXL2) have been described in several human malformations ${ }^{10,11}$. In each of these cases, both the normal and expanded alanine tracts range in a similar size, suggesting a common underlying mechanism. In the case of HOXD13 and ZIC2, the expansions are not just loss-of-function mutations but are responsible for a dominant negative effect ${ }^{12,13}$. In the case of $P H O X 2 B$, a loss-of-function mutation with lower penetrance for the enteric nervous system anomalies is a possibility, as we found frameshift mutations in two individuals. Assuming that the protein is stable, however, the homeodomain is preserved in 
both cases. As far as the ocular phenotype is concerned, one can speculate that PHOX2B mutant protein products exert a dominant negative effect on PHOX2A, considering the overlapping features observed in CCHS and congenital fibrosis of the extra ocular muscle type 2 (CFEOM2) resulting from homozygous mutations of the gene PHOX2A (ref. 14) Indeed, it has been shown that the third and fourth motor nuclei express both Phox $2 \mathrm{~A}$ and Phox $2 \mathrm{~b}$ in mice and are Phox2a-dependent ${ }^{8}$. The mechanism for a putative involvement of $P H O X 2 B$ in isolated neural-crest tumors is undefined at present, but it is worth mentioning that Phox $2 b$ has been shown to regulate neuronal cell cycle ${ }^{15}$.

Little is known regarding the bases of ventilatory control anomalies in CCHS It has been speculated that the disease involves a defect in the integration by the nucleus of the solitary tract and interneurons of the inputs from the central $\mathrm{CO}_{2} / \mathrm{pH}$-sensitive chemoreceptors (medulla oblongata) and the peripheral $\mathrm{O}_{2}, \mathrm{CO}_{2}$ and $\mathrm{pH}$-sensitive chemoreceptors in the carotid bodies ${ }^{1}$. Notably, several of these structures express Phox $2 b$ in mice ${ }^{8,9}$ and humans (this study) and fail to form or degenerate in Phox $2 \mathrm{~b}^{-/-}$ mouse mutants (refs. 8,9 and J.-F. Brunet, pers. comm.). So far, no phenotype has been reported in Phox $2 \mathrm{~b}^{+/-}$mice. Our mutation and expression studies strongly support the view that $P H O X 2 B$ is a master gene for the formation and/or function of the neuronal network for autonomous control of ventilation and further suggests that $P H O X 2 B$ mutations trigger a wide spectrum of ANS disorders ranging from dysgenetic malformations to tumor predisposition.

Note: Supplementary information is available on the Nature Genetics website.

\section{Acknowledgments}

We thank the individuals with CCHS, their families and the Association Française du Syndrome d'Ondine who participated in this study, J.-F. Brunet and C. Goridis for helpful comments and discussions and G. Mattéi for technical help. This study was supported by grants from the European Community, Association Française contre les Myopathies-INSERM (Maladies Rares) and Hoechst-Marion-Roussel. B.L. is a recipient of a Sanofi-Synthelabo grant.

\section{Competing interests statement}

The authors declare that they have no competing financial interests.

Jeanne Amiel ${ }^{1}$, Béatrice Laudier ${ }^{1}$, Tania Attié-Bitach ${ }^{1}$, Ha Trang ${ }^{2}$, Loïc de Pontual ${ }^{1}$, Blanca Gener ${ }^{3}$, Delphine Trochet $^{1}$, Heather Etchevers ${ }^{1}$, Pierre Ray ${ }^{1}$,
Michel Simonneau², Michel Vekemans ${ }^{1}$, Arnold Munnich ${ }^{1}$, Claude Gaultier ${ }^{2}$ \& Stanislas Lyonnet ${ }^{1}$

${ }^{1}$ Unité de Recherches sur les Handicaps Génétiques de I'Enfant INSERM U-393, et Département de Génétique, Hôpital NeckerEnfants Malades, 149, rue de Sèvres, 75743 Paris Cedex 15, France. ${ }^{2}$ Service de Physiologie CIC INSERM 9202, et Equipe INSERM E9935, Hôpital Robert Debré, Paris, France. ${ }^{3}$ Clinica Materno-Infantil, Hospital de Cruces, 48903 Barakaldo, Spain. Correspondence should be addressed to J.A. (e-mail: amiel@necker.fr).

Received 31 October 2002; accepted 20 February 2003.

1 Gozal, D. Pediatr. Pulmonol. 26, 273-282 (1998). 2. Khalifa, M.M., Flavin, M.A. \& Wherrett, B.A. J. Pediatr. 113, 853-855 (1988).

3. Sritippayawan, S. et al. Am. J. Respir. Crit. Care Med. 166, 367-369 (2002)

4. Weese-Mayer, D.E., Silvestri, J.M., Marazita, M.L. \& Hoo, J.J. Am. J. Med. Genet. 47, 360-367 (1993).

5. Amiel, J. et al. Am. J. Hum. Genet. 62, 715-717 (1998).

6. Bolk, S. et al. Nat. Genet. 13, 395-396 (1996).

7. Weese-Mayer, D.E., Bolk, S., Silvestri, J.M. \& Chakravarti, A. Am. J. Med. Genet. 107, 306-310 (2002).

8. Brunet, J.F. \& Pattyn, A. Curr. Opin. Genet. Dev. 12, 435-440 (2002).

9. Pattyn, A., Morin, X., Cremer, H., Goridis, C. \& Brunet, J.F. Nature 399, 366-370 (1999).

10. Goodman, F.R. \& Scambler, P.J. Clin. Genet. 59, 1-11 (2001).

11. Stromme, P. et al. Nat. Genet. 30, 441-445 (2002).

12. Bruneau, S., Johnson, K.R., Yamamoto, M., Kuroiwa, A. \& Duboule, D. Dev. Biol. 237, 345-353

13. Brown, S.A., Abigani, M. \& Brown, L.Y. Am. J. Hum. Genet. 71 Supp. 166 (2002)

14. Nakano, M. et al. Nat. Genet. 29, 315-320 (2001).

15. Dubreuil, V., Hirsch, M.R., Pattyn, A., Brunet, J.F. \& Goridis, C. Development 127, 5191-5201 (2000). 